\title{
High Incidence of Abnormal Circadian Blood Pressure Profiles in Patients on Steroid Replacement Therapy due to Secondary Adrenal Insufficiency and Congenital Adrenal Hyperplasia without Overt Hypertension - Initial Results
}

\section{Malgorzata Wojcik ${ }^{1,2 \star}$, Dominika Janus ${ }^{1,2}$, Karolina Poplawska ${ }^{3}$, Katarzyna Tyrawa², Agata Zygmunt-Gorska² and Jerzy B Starzyk ${ }^{1,2}$}

${ }^{1}$ Department of Pediatric and Adolescent Endocrinology, Chair of Pediatrics, Polish-American Institute of Pediatrics, Jagiellonian University Medical College, Poland ${ }^{2}$ Department of Pediatric and Adolescent Endocrinology, Children's University Hospital in Krakow, Poland

${ }^{3}$ Department of Pediatrics, Children's University Hospital in Krakow, Poland

\begin{abstract}
Patients on steroid replacement therapy are at an increased risk of cardiovascular complications owing to the fact that disruptions in the cortisol diurnal rhythm may affect the blood pressure (BP) profile.

Aim: To evaluate the circadian BP profiles of patients with secondary adrenal insufficiency (SAI) and congenital adrenal hyperplasia $(\mathrm{CAH})$ on steroid replacement therapy and to compare BP profiles of patients receiving hydrocortisone $(\mathrm{HC})$ in different dosing schedules.

Methods: The study included 33 patients: $15 \mathrm{SAl}$ and $18 \mathrm{CAH}$ (mean age 13.2 years $95 \mathrm{Cl} 11.3-15.1$ ). There were no patients with previously diagnosed overt hypertension. Patients with SAI received a mean of $7.39 \mathrm{mg} / \mathrm{m}^{2}$ of $\mathrm{HC}$ in 3 daily doses (in the morning (M) $50 \%$, in the afternoon (A) $25 \%$, in the evening (E) $25 \%$ ), CAH patients $17.9 \mathrm{mg} / \mathrm{m}^{2}$ of HC in the following dosing schedules: 5 patients in 3 equal doses, 7 patients received $\mathrm{M}: 40 \% \mathrm{~A}: 40 \% \mathrm{E}: 20 \%$, the remaining 6 patients had the same dosing schedule as patients with SAI. Fludrocortisone (FC) was given to 13 patients with $\mathrm{CAH}$ in 2 equal daily doses. The total dose of HC/FC as well as the dosing schedule of $\mathrm{HC}$ was adjusted individually based on clinical and biochemical outcomes. Standard 24-hour BP monitoring (ABPM) was performed using an Ambulatory BP Monitor (Space labs 90217, USA).

Results: The majority of the patients (almost $70 \% \mathrm{SAI}, 80 \% \mathrm{CAH}$ ) presented with an abnormal 24-hour BP profile. There were no significant differences in ABPM results between SAI and CAH patients, and no differences between $\mathrm{CAH}$ patients treated with and without FC. There was no correlation between $\mathrm{HC}$ and $\mathrm{FC}$ doses $\left[\mathrm{mg} / \mathrm{m}^{2}\right]$ and $\mathrm{ABPM}$ results except that mean night SBP values increased with greater $\mathrm{HC}$ doses $(r=0.51, p<0.05)$. Among the CAH group the highest percentage of abnormal ABPM results was observed in patients who received $\mathrm{HC}$ in doses: M: $50 \%$ A: $25 \%$ and $\mathrm{E}: 25 \%$, the most favorable BP profile was observed in patients with dosing schedule: M: $40 \%, A: 40 \%$, E: $20 \%$. However there were no significant differences between patients with different treatment protocols, the results suggest that observed disruptions of the BP profile could be related to the $\mathrm{HC}$ dosing schedule.

Conclusions: The incidence of abnormal BP profiles in patients on steroid replacement therapy due to SAI and $\mathrm{CAH}$ without overt hypertension is high. The disruptions of the BP profiles are not associated with the dose of $\mathrm{HC}$ or FC. The abnormal BP profiles in patients with SAI or CAH may be related to the HC dosing schedule. 24-hour ABPM seems to be a useful, non-invasive and safe method for the monitoring of $\mathrm{HC}$ and FC replacement therapy in patients with adrenal insufficiency. Further investigations in the larger groups of patients are needed.
\end{abstract}

Keywords: Congenital adrenal hyperplasia; Secondary adrenal insufficiency; Hypertension; ABPM; Cortisol replacement

Abbreviations: ABPM: Ambulatory Blood Pressure Monitoring; ACTH: Adrenocorticotropic Hormone; ADH: Anti-diuretic Hormone; BP: Blood Pressure; CAH: Congenital Adrenal Hyperplasia; dDBP: Mean Day-Time Diastolic BP; dMAP: Mean Day-Time Arterial Pressure; dSBP: Mean Day-Time Systolic BP; FC: Fludrocortisone; FSH: Follicle-Stimulating Hormone; GH: Growth Hormone; HC: Hydrocortisone; LH: Luteinizing Hormone; MAP: Mean Arterial Pressure; nDBP: Mean Night-Time Diastolic BP; nMAP: Mean NightTime Arterial Pressure; nSBP: Mean Night-Time Systolic BP; SAI: Secondary Adrenal Insufficiency; TSH: Thyroid-Stimulating Hormone

\section{Introduction}

Serum cortisol levels and blood pressure (BP) both have welldocumented circadian rhythms. Normally, cortisol levels peak in the early morning and drop to their lowest concentration at night $[1,2]$. The BP circadian rhythm is typically characterized by minor fluctuations throughout the day and night, with an overall dipping that occurs during the night. In normotensive subjects there is typically a $10 \%$ to $20 \%$ reduction in BP during sleep [3-5]. Most studies that examine cortisol and BP rhythms tend to look at them in isolation, which could

*Corresponding author: Malgorzata Wojcik, Department of Pediatric and Adolescent Endocrinology, Chair of Pediatrics, Polish-American Institute of Pediatrics, Jagiellonian University Medical College, Wielicka St. 265, $30-$ 663 Krakow, Poland, Tel: +48 1265812 77; Fax: +48 1265810 05; E-mail: wojcik.gosia@gmail.com

Received July 15, 2013; Accepted August 28, 2013; Published August 31, 2013

Citation: Wojcik M, Janus D, Poplawska K, Tyrawa K, Zygmunt-Gorska A, et al. (2013) High Incidence of Abnormal Circadian Blood Pressure Profiles in Patients on Steroid Replacement Therapy due to Secondary Adrenal Insufficiency and Congenital Adrenal Hyperplasia without Overt Hypertension - Initial Results. J Steroids Hormon Sci S12: 005. doi:10.4172/2157-7536.S12-005

Copyright: ( 2013 Wojcik M, et al. This is an open-access article distributed under the terms of the Creative Commons Attribution License, which permits unrestricted use, distribution, and reproduction in any medium, provided the original author and source are credited. 
Citation: Wojcik M, Janus D, Poplawska K, Tyrawa K, Zygmunt-Gorska A, et al. (2013) High Incidence of Abnormal Circadian Blood Pressure Profiles in Patients on Steroid Replacement Therapy due to Secondary Adrenal Insufficiency and Congenital Adrenal Hyperplasia without Overt Hypertension - Initial Results. J Steroids Hormon Sci S12: 005. doi:10.4172/2157-7536.S12-005

obscure the potential links between them. There are only a few studies that confirm an association between cortisol levels and BP fluctuations [2,6-9]. An elegant study performed by Holt-Lunstad and Steffen in 302 healthy adult volunteers revealed that a decreased diurnal variation in cortisol levels was associated with a decreased diurnal variation in $\mathrm{BP}$, whereas normal cortisol levels were a significant and independent predictor of normal BP dipping [2]. An abnormal BP circadian rhythm, and in particular a non- dipping phenomenon is associated with increased health risks including left ventricular hypertrophy [10-13], cerebrovascular stroke [14], cardiovascular morbidity [12], kidney damage [14], and increased mortality $[15,16]$. Thus, disrupted circadian cortisol and in consequence BP rhythms may have important long-term health implications. All patients with adrenal insufficiency on life-long hydrocortisone (HC) replacement therapy are at risk of both cortisol rhythm and BP profile disruption and their sequelae. Thus the HC supplementation regimen could be crucial. Under normal circumstances the secretion of cortisol and adrenal androgens is under the dominant control of pituitary ACTH. In contrast, aldosterone, which has a predominantly mineralocorticoid function, is primarily regulated by the renin-angiotensin system. It is also additionally influenced by sodium, potassium, dopamine, and serotonin levels [17].

Secondary adrenal insufficiency (SAI) (central hypoadrenalism) characterized by a deficit of ACTH is commonly found in patients with multihormonal pituitary deficiency (GH, TSH, LH, FSH, ADH) [17]. The most common cause of congenital adrenal hyperplasia $(\mathrm{CAH})$ is 21-hydroxylase deficiency [18], which leads to a decrease in cortisol and aldosterone synthesis (in 75\% of cases). Lack of cortisol leads to an increase in ACTH and stimulation of the adrenal cortex, with accumulation of cortisol precursors that are shunted to the sex hormone pathway [18].

The current treatment of SAI and CAH consists of the administration of glucocorticoids, and in the case of CAH mineralocorticoids as well (Fludrocortisone, FC) if necessary. The goal of treatment in both diseases is replacement of cortisol to prevent adrenal crisis, and to suppress the abnormal secretion of adrenal androgens in CAH $[17,18]$. Hydrocortisone (HC) is considered the drug of choice in these two cases. For the reason that modern techniques which measure cortisol production rates have suggested that the mean total daily production is $10 \mathrm{mg} / \mathrm{m}^{2}$, that dose of HC is recommended $[17,19,20]$. In order for it to correlate with the circadian rhythm, the dose should be administered three times a day, usually $50 \%$ of the dose in the morning, $25 \%$ around lunchtime, and $25 \%$ in the early evening [17,20,21]. In order to initially decrease markedly elevated sex hormone levels in CAH patients during infancy, doses of up to $25 \mathrm{mg} / \mathrm{m}^{2}$ of $\mathrm{HC}$ may be required, however in growing children a typical dosing schedule is $10-15 \mathrm{mg} / \mathrm{m}^{2}$ three times a day. After achieving adult height, patients are often switched to longer-acting glucocorticoid preparations that can be given once or twice a day to maximize convenience and androgen synthesis inhibition. Some authors recommend using a reverse circadian pattern of $\mathrm{HC}$ replacement therapy to optimize androgen suppression, because glucocorticoid given before bedtime suppresses the ACTH night-time surge [22-24]. Others advocate giving the highest glucocorticoid dose in the morning or even waking the patient up at 3:00 am in an attempt to approximate the normal physiological timing of cortisol secretion [2528]. Nevertheless no current glucocorticoid therapy is able to mimic normal cortisol circadian rhythms, and in consequence BP rhythms which may contribute to suboptimal clinical outcomes. The patients on steroid replacement are undoubtedly at higher (in comparison to general population) risk of overt hypertension. Moreover, even if they present with normal results of the casual BP measurements, they may have disruptions of the circadian BP rhythm [2,7-9]. To date, investigators have failed to determine unequivocally what is the frequency of such disorders and what are the main determinants and risk factors $[2,7-9]$.

\section{The Aim of the Study}

To evaluate daily BP profiles in patients with SAI and CAH on $\mathrm{HC}$ replacement therapy $[1,2]$. To compare $\mathrm{BP}$ profiles in patients with $\mathrm{CAH}$ treated with $\mathrm{HC}$ in three different dosing schedules.

\section{Patients and Methods}

The studied group of this pilot study consisted of 33 patients with adrenal insufficiency, of which 15 had secondary adrenal insufficiency ( 8 girls and 7 boys) and 18 (11 girls and 7 boys) congenital adrenal hyperplasia due to 21 hydroxylase deficiency (the diagnosis was established based on the results of urinary steroid profile analysis using gas chromatography/mass spectrometry).

The mean age was 13.2 years with a confidence interval of $95 \mathrm{CI}$ (11.3-15.1)

The exclusion criteria were: presence of previously diagnosed overt hypertension, anti-hypertensive treatment and other endocrinological disorders except secondary thyroid insufficiency and growth hormone deficiency on hormonal replacement therapy.

In the group of patients with secondary adrenal insufficiency all were affected by multiple pituitary insufficiency, 12 had congenital defects of the pituitary gland and 3 acquired pituitary insufficiency due to pituitary tumors.

There were no significant differences with regard to age and auxological parameters (Table 1).

The daily hydrocortisone dose was significantly higher in the $\mathrm{CAH}$ group (13 patients were also treated with FC). In all patients with SAI, hydrocortisone was administered in 3 daily doses: $50 \%$ of the daily dose was given in the morning (M), $25 \%$ in the afternoon (A) and $25 \%$ in the evening (E). In the $\mathrm{CAH}$ group, 5 patients received hydrocortisone in 3 equal doses, 7 received: M: 40\%, A: 40\%, E: 20\%, 6 patients had a dosing schedule similar the one in patients with SAI (M: 50\%, A: $25 \%$, E: $25 \%)$. The total dose of $\mathrm{HC}$ as well as the dosing schedule was adjusted individually based on clinical and biochemical outcomes

\begin{tabular}{|c|c|c|c|}
\hline Parameters & Secondary adrenal insufficiency mean $[95 \% \mathrm{CI}]$ & Congenital adrenal hyperplasia mean $[95 \% \mathrm{Cl}]$ & p \\
\hline $\mathrm{N}$ & 15 & 18 & - \\
\hline Age [years] & $13.2[11.3-15.1]$ & $11[9.2-12.7]$ & 0.07 \\
\hline Height [SDS] & $-0.49[-1.25-0.26]$ & $-0.52[-1.3-0.25]$ & 0.79 \\
\hline BMI [SDS] & $0.04[-0.5-0.6]$ & $0.21[-0.4-0.8]$ & 0.61 \\
\hline Hydrocortisone daily dose $\mathrm{mg} / \mathrm{m}^{2}$ & $7.39[6.1-8.6]$ & $17.9[15.4-20.3]$ & 0.002 \\
\hline Fludrocortisone daily dose $\mathrm{mg} / \mathrm{m}^{2}$ & - & $0.03[0.01-0.04]$ & - \\
\hline
\end{tabular}

Table 1: Characteristics of the studied groups: secondary adrenal insufficiency (SAI) and congenital adrenal hyperplasia (CAH). 
Citation: Wojcik M, Janus D, Poplawska K, Tyrawa K, Zygmunt-Gorska A, et al. (2013) High Incidence of Abnormal Circadian Blood Pressure Profiles in Patients on Steroid Replacement Therapy due to Secondary Adrenal Insufficiency and Congenital Adrenal Hyperplasia without Overt Hypertension - Initial Results. J Steroids Hormon Sci S12: 005. doi:10.4172/2157-7536.S12-005

such as: growth velocity, body weight, bone age, blood pressure, 17-hydroxyprogesteron levels in the blood and 17-ketosteroid levels in the urine. Fludrocortisone (FC) was given in 2 daily doses, the total dose was adjusted based on plasma renin activity and BP.

Body weight and height were measured to the nearest $0.1 \mathrm{~kg}$ and $0.1 \mathrm{~cm}$, respectively, using a stadiometer (Harpenden, UK) and a balanced scale. As the standard of reference normal values from the local population were used [29].

24-hour BP monitoring was performed using an Ambulatory BP Monitor (Space labs 90217, USA), with a cuff which was the same size as the one used to measure casual blood pressure. It was set to take a reading every 15 minutes (day 6 a.m.-10:59 p.m.), and every 30 minutes (night 11:00 p.m.-5:59 a.m.). The monitoring was performed in a standard setting, patients went to sleep at 9-10 p.m. and got up at 6-7 a.m. Sleep and wake periods were established based on a diary completed by the child's parents. Recordings with at least $70 \%$ valid readings and at least one reading every hour were considered for the analysis. The following parameters were analyzed: mean 24-h systolic (SBP), diastolic (DBP), and mean arterial pressure (MAP), mean day-time systolic (dSBP), diastolic (dDBP), and MAP (dMAP), mean night-time systolic (nSBP), diastolic (nDBP), and MAP (nMAP). Blood pressure load was calculated separately for the awake and asleep periods. BP load was defined as the percentage of valid BP measurements above a set threshold $\left(95^{\text {th }}\right.$ percentile for sex and the height) value $[4,5]$. Loads in excess of $30 \%$ were considered elevated. Loads in excess of $50 \%$ were considered severely elevated. The calculation of nocturnal dipping was based on a formula by the American Heart Association: [(dSBP$\mathrm{nSBP}) / \mathrm{dSBP}] \times 100$. Normal dipping was defined as $\mathrm{a} \geq 10 \%$ decline in $\mathrm{BP}[3,5]$.

In order to compare the two groups the two-sided Mann-Whitney U-test and ANOVA tests were used. Spearman $\rho$ was used to measure the strength of association between pairs of variables. The level of significance was set at $\mathrm{p}<0.05$. Calculations were performed using the STATISTICA 10.0 PL soft ware (Poland).

\section{Results}

The majority of the patients presented with abnormal 24-hour BP profile: almost $70 \%$ with SAI $80 \%$ with CAH. The patients with normal $\mathrm{BP}$ profiles were significantly younger in comparison to individuals with BP profile abnormalities (mean 9.6 vs. 12.8 years).

There were no significant differences in ABPM results between SAI and CAH patients. The mean values of the $24 \mathrm{~h}$ SBP, day time SBP and night time SBP were slightly higher in the CAH group, however the differences were not significant (SBP: 108.8 vs. $112.2 \mathrm{mmHg}$, dSBP: 111.7 vs. $114.2 \mathrm{mmHg}$, nSBP: 100.6 vs. $105.2 \mathrm{mmHg}$ in SAI and $\mathrm{CAH}$ groups respectively). The mean 24-hour SBP loads were also comparable: an elevated SBP load was observed in $20 \%$ of patients with SAI and in $17 \%$ of CAH patients (in $6 \%$ of CAH patients elevation was severe). Day time SBP load was elevated in $20 \%$ of SAI and $12 \%$ of $\mathrm{CAH}$ patients (severely elevated in 6\% CAH patients). Interestingly, the night time SBP seems to be more affected in CAH group since the night time SBP load was elevated in $26 \%$ (13\% seriously elevated) of SAI, and 34\% (28 seriously elevated) of CAH patients.

There were also no significant differences among 24h DBP, day time DBP and night time DBP mean values between SAI and CAH patients (66.5 vs. $67.2 \mathrm{mmHg}$, 69.7 vs. $69.3 \mathrm{mmHg}$, 56.6 vs. $59.5 \mathrm{mmHg}$ respectively). An elevated 24 -h DBP load was observed in $13 \%$ of SAI and in $11 \%$ of CAH patients. $27 \%$ of SAI patients presented an elevated day time DBP load (severely elevated in 7\%), but interestingly it was not observed in any patient with CAH. Night time DBP load elevation was present in $27 \%$ of SAI patients (severely elevated in $7 \%$ ), and in $23 \%$ of CAH patients (severely elevated in $17 \%$ ).

The mean values of the $24 \mathrm{~h}$ MAP, day time MAP and night time MAP were comparable in both groups: $24 \mathrm{~h}$ MAP 81.5 vs. $82.8 \mathrm{mmHg}$, dMAP 84 vs. $84.6 \mathrm{mmHg}$, nMAP 71.5 vs. $75.8 \mathrm{mmHg}$ in SAI and CAH patients respectively.

In 21 patients $8(53 \%)$ with SAI and 13 (72\%) with CAH (of which 11 patients were on $\mathrm{FC}$ ) there was no significant night time dip. Nevertheless there was no significant difference in the mean value of the nighttime dip between SAI and CAH patients (9.91 vs. $7.88 \%$ ). There were no significant differences in ABPM results between $\mathrm{CAH}$ patients treated with or without FC (Table 2).

There was no correlation between $\mathrm{HC}$ and $\mathrm{FC}$ doses $\left[\mathrm{mg} / \mathrm{m}^{2}\right]$ and 24 hour ABPM results except that mean night SBP values increased with greater $\mathrm{HC}$ doses $(\mathrm{r}=0.51, \mathrm{p}<0.05)$.

Among the $\mathrm{CAH}$ group the highest percentage of abnormal ABPM results was observed in patients who received $\mathrm{HC}$ in doses: $\mathrm{M}: 50 \% \mathrm{~A}$ : $25 \%$ and $\mathrm{E}: 25 \%$, the most favorable BP profile was observed in patients with dosing schedule: M: 40\%, A: 40\%, E: 20\% (Table 3). However there were no significant differences between patients with different treatment protocols, the results suggest that observed disruptions of the BP profile could be related to the HC dosing schedule.

\section{Discussion}

The circadian rhythm of blood pressure is maintained within normal limits through the interplay of various mechanisms including the secretion of cortisol and aldosterone [1,2]. Unfortunately when these hormones are exogenously administered they are not under the regulatory feedback mechanisms that maintain BP homeostasis. The results of the present study show that long term $\mathrm{HC}$ and/or FC replacement therapy do not seem to be associated with overt hypertension in young people with SAI or CAH when using casual $\mathrm{BP}$ references. This observation is in agreement with previously published data [7]. Even though no single case of overt hypertension was noted, almost $70 \%$ of SAI and $80 \%$ of $\mathrm{CAH}$ patients presented with

\begin{tabular}{|l|c|c|c|}
\hline & Fludrocortisone & no fludrocortisone & $\mathbf{p}$ \\
\hline $\mathrm{HC}$ dose $\left[\mathrm{mg} / \mathrm{m}^{2}\right]$ & 17.7 & 18.3 & 0.55 \\
\hline mean 24h SBP $(\mathrm{mmHg})$ & 111.1 & 115.2 & 0.3 \\
\hline mean 24h DBP $(\mathrm{mmHg})$ & 65.8 & 70.8 & 0.1 \\
\hline mean 24h MAP $(\mathrm{mmHg})$ & 81.6 & 85.8 & 0.27 \\
\hline mean dSBP $(\mathrm{mmHg})$ & 113.1 & 117.4 & 0.12 \\
\hline mean dDBP $(\mathrm{mmHg})$ & 68 & 72.8 & 0.13 \\
\hline mean dMAP $(\mathrm{mmHg})$ & 83.6 & 87.4 & 0.13 \\
\hline mean nSBP $(\mathrm{mmHg})$ & 105 & 106 & 0.8 \\
\hline mean nDBP $(\mathrm{mmHg})$ & 58.3 & 62.6 & 0.2 \\
\hline mean nMAP $(\mathrm{mmHg})$ & 75 & 77.8 & 0.3 \\
\hline $24 \mathrm{~h} \mathrm{SBP} \mathrm{load}(\%)$ & 14.3 & 19.7 & 0.4 \\
\hline dSBP load $(\%)$ & 12 & 16.9 & 0.3 \\
\hline nSBP load $(\%)$ & 22 & 27.1 & 0.9 \\
\hline $24 \mathrm{~h}$ DBP load $(\%)$ & 10.7 & 17.8 & 0.1 \\
\hline dDBP load $(\%)$ & 9.5 & 13.9 & 0.2 \\
\hline nDBP load $(\%)$ & 15.4 & 31 & 0.1 \\
\hline night time dip $(\%)$ & 7.1 & 9.6 & 0.5 \\
\hline
\end{tabular}

Table 2: Outcome variables in patients with $\mathrm{CAH}$ with and without fludrocortisone replacement therapy. 
Citation: Wojcik M, Janus D, Poplawska K, Tyrawa K, Zygmunt-Gorska A, et al. (2013) High Incidence of Abnormal Circadian Blood Pressure Profiles in Patients on Steroid Replacement Therapy due to Secondary Adrenal Insufficiency and Congenital Adrenal Hyperplasia without Overt Hypertension - Initial Results. J Steroids Hormon Sci S12: 005. doi:10.4172/2157-7536.S12-005

Page 4 of 5

\begin{tabular}{|c|c|c|c|c|c|}
\hline Parameters & & Three equal daily doses & $40+40+20 \%$ & $50+25+25 \%$ & $\mathbf{P}$ \\
\hline \multirow{3}{*}{$24 \mathrm{~h}$ SBP } & mean $[\mathrm{mmHg}]$ & 110 & 112.1 & 114.1 & 0.52 \\
\hline & load [\%] & 13.9 & 8.6 & 25.9 & 0.42 \\
\hline & percentage of abnormal load values [\%] & 0 & 0 & 33 & \\
\hline \multirow{3}{*}{ 24h DBP } & mean $[\mathrm{mmHg}]$ & 66.2 & 66 & 68.2 & 0.78 \\
\hline & Ioad [\%] & 12.4 & 9.3 & 16.9 & 0.6 \\
\hline & percentage of abnormal load values [\%] & 0 & 0 & 33 & \\
\hline \multirow{3}{*}{ dSBP } & mean $[\mathrm{mmHg}]$ & 112.6 & 114.1 & 115.8 & 0.8 \\
\hline & load [\%] & 10.4 & 7.6 & 22.6 & 0.38 \\
\hline & percentage of abnormal load values [\%] & 0 & 0 & 33 & \\
\hline \multirow{3}{*}{ dDBP } & mean $[\mathrm{mmHg}]$ & 68.8 & 69.4 & 69.7 & 0.9 \\
\hline & Ioad [\%] & 9.38 & 8.9 & 14 & 0.8 \\
\hline & percentage of abnormal load values [\%] & 0 & 0 & 0 & \\
\hline \multirow{3}{*}{ nSBP } & mean $[\mathrm{mmHg}]$ & 102.4 & 104 & 109 & 0.55 \\
\hline & load [\%] & 23.94 & 9.6 & 39.3 & 0.26 \\
\hline & percentage of abnormal load values [\%] & 20 & 14 & 50 & \\
\hline \multirow{3}{*}{ nDBP } & mean $[\mathrm{mmHg}]$ & 59.2 & 57.9 & 62 & 0.45 \\
\hline & load [\%] & 20.8 & 10.9 & 29.3 & 0.6 \\
\hline & percentage of abnormal load values [\%] & 20 & 0 & 30 & \\
\hline \multirow{2}{*}{ Night time dip } & [\%] & 9.05 & 8.8 & 5.7 & 0.18 \\
\hline & percentage of abnormal load values [\%] & 80 & 57 & 100 & \\
\hline
\end{tabular}

Table 3: Outcome variables in patients with $\mathrm{CAH}$ with different $\mathrm{HC}$ dosing schedules

an abnormal 24-hour BP profile. The results of the study shows, those casual, ambulatory BP measurements are not a sufficient tool for the monitoring of long-term cardiovascular risk factors in such patients, because adrenocortical insufficiency and subsequent replacement therapy are not usually associated with overt hypertension, but have often been shown to account for a loss of circadian blood pressure rhythm. Due to the fact that cortisol and BP circadian rhythms are closely linked, 24-hour BP monitoring may be a useful additional tool for the non-invasive monitoring of replacement therapy.

Our results show that the total daily dose of $\mathrm{HC}$ was not associated with 24-hour BP profile parameters, except mean night time SBP. The lack of such an association was also showed in the study performed by Ubertini et al. [8]. Our results also show that there was no significant impact of the FC, since there were no significant differences between patients with $\mathrm{CAH}$ receiving only $\mathrm{HC}$ and combined $\mathrm{HC}+\mathrm{FC}$ therapy. However in the group of patients receiving FC the incidence of decreased night time dip was higher compared to the group receiving $\mathrm{HC}$ alone, but there were no significant difference in the mean values of night time dip between these two groups. Interestingly, decreased night time dip was also observed in the majority (53\%) of patients with SAI.

A more important factor which can modulate the circadian variation of BP might be the regimen of HC administration. Only a few studies have been dedicated to the analysis of the relationship between the HC administration model and BP profiles in such groups of patients [6,9]. In these studies the administration of a higher morning dose of $\mathrm{HC}$ was associated with a more physiologic BP rhythm and preservation of night time dipping. The authors suggested that patients with SAI on $\mathrm{HC}$ replacement therapy could maintain an approximately normal BP rhythm. This has not been confirmed in the present study. Only $46 \%$ of SAI patients presented night time dip $\geq 10 \%$. The problem is even more complicated in patients with CAH. A few recent studies, investigating $\mathrm{BP}$ profiles in $\mathrm{CAH}$ patients, have reported conflicting results. Even though Ubertini et al. showed no significant differences between $\mathrm{CAH}$ patients and the general population concerning BP profiles [8], other authors indicated a higher prevalence of abnormalities, especially a decrease in night time dip in these patients $[30,31]$. One study with $11 \mathrm{CAH}$ participants showed that a more physiological administration of $\mathrm{HC}$ with a higher morning dose was associated with a more physiological BP profile, with greater night time dip, and not associated with poor biochemical parameters of control of the disease [6].

Our results show that the risk of an abnormal BP profile is present in as many as $80 \%$ of $\mathrm{CAH}$ patients, irrespective of $\mathrm{HC}$ replacement schedule and additional treatment with FC. Nevertheless the most favorable profile was observed in patients receiving $40 \%$ of their daily dose in the morning, $40 \%$ in the afternoon and $20 \%$ in the evening. This novel observation needs further examination in larger groups of patients.

\section{Conclusions}

The incidence of abnormal BP profiles in patients on steroid replacement therapy due to SAI and CAH without overt hypertension is high. The disruptions of the BP profiles are not associated with the dose of $\mathrm{HC}$ neither with FC treatment (in CAH patients). The abnormal $\mathrm{BP}$ profiles in patients with SAI or CAH might be related to the HC dosing schedule. 24-hour ABPM seems to be a useful, non-invasive and safe method for the monitoring of $\mathrm{HC}$ and $\mathrm{FC}$ replacement therapy in patients with adrenal insufficiency. Further investigations in the larger groups of patients are needed.

\section{References}

1. Kalsbeek A, Fliers E (2013) Daily regulation of hormone profiles. Handb Exp Pharmacol: 185-226.

2. Holt-Lunstad J, Steffen PR (2007) Diurnal cortisol variation is associated with nocturnal blood pressure dipping. Psychosom Med 69: 339-343.

3. Urbina E, Alpert B, Flynn J, Hayman L, Harshfield GA, et al. (2008) Ambulatory blood pressure monitoring in children and adolescents: recommendations for standard assessment: a scientific statement from the American Heart Association Atherosclerosis, Hypertension, and Obesity in Youth Committee of the Council on Cardiovascular Disease in the young and the council for high blood pressure research. Hypertension 52: 433-451.

4. Wühl E, Witte K, Soergel M, Mehls O, Schaefer F; German Working Group on Pediatric Hypertension (2002) Distribution of 24-h ambulatory blood pressure in children: normalized reference values and role of body dimensions. J Hypertens 20: 1995-2007.

5. National High Blood Pressure Education Program Working Group on High 
Citation: Wojcik M, Janus D, Poplawska K, Tyrawa K, Zygmunt-Gorska A, et al. (2013) High Incidence of Abnormal Circadian Blood Pressure Profiles in Patients on Steroid Replacement Therapy due to Secondary Adrenal Insufficiency and Congenital Adrenal Hyperplasia without Overt Hypertension - Initial Results. J Steroids Hormon Sci S12: 005. doi:10.4172/2157-7536.S12-005

Page 5 of 5

Blood Pressure in Children and Adolescents (2004) The fourth report on the diagnosis, evaluation, and treatment of high blood pressure in children and adolescents. Pediatrics 114: 555-576.

6. Liivak K, Tillmann V (2009) 24-hour blood pressure profiles in children with congenital adrenal hyperplasia on two different hydrocortisone treatment regimens. J Pediatr Endocrinol Metab 22: 511-517.

7. de Silva KS, Kanumakala S, Brown JJ, Jones CL, Warne GL (2004) 24 hour ambulatory blood pressure profile in patients with congenital adrenal hyperplasia--a preliminary report. J Pediatr Endocrinol Metab 17: 1089-1095.

8. Ubertini G, Bizzarri C, Grossi A, Gimigliano F, Ravà L, et al. (2009) Blood Pressure and Left Ventricular Characteristics in Young Patients with Classical Congenital Adrenal Hyperplasia due to 21-Hydroxylase Deficiency. Int J Pediatr Endocrinol 2009: 383610.

9. Matsumura K, Abe I, Fukuhara M, Fujii K, Ohya Y, et al. (1994) Modulation of circadian rhythm of blood pressure by cortisol in patients with hypopituitarism. Clin Exp Hypertens 16: 55-66.

10. Fumo MT, Teeger S, Lang RM, Bednarz J, Sareli P, et al. (1992) Diurnal blood pressure variation and cardiac mass in American blacks and whites and South African blacks. Am J Hypertens 5: 111-116.

11. Sihm I, Schroeder AP, Aalkjaer C, Holm M, Mørn B, et al. (1995) The relation between peripheral vascular structure, left ventricular hypertrophy, and ambulatory blood pressure in essential hypertension. Am J Hypertens 8: 987 996

12. Verdecchia P, Schillaci G, Borgioni C, Ciucci A, Sacchi N, et al. (1995) Gender day-night blood pressure changes, and left ventricular mass in essential hypertension. Dippers and peakers. Am J Hypertens 8: 193-196.

13. Kario K, Matsuo T, Kobayashi H, Imiya M, Matsuo M, et al. (1996) Nocturnal fall of blood pressure and silent cerebrovascular damage in elderly hypertensive patients. Advanced silent cerebrovascular damage in extreme dippers. Hypertension 27: 130-135.

14. Timio M, Venanzi S, Lolli S, Lippi G, Verdura C, et al. (1995) "Non-dipper" hypertensive patients and progressive renal insufficiency: a 3-year longitudinal study. Clin Nephrol 43: 382-387.

15. Ohkubo T, Imai Y, Tsuji I, Nagai K, Watanabe N, et al. (1997) Relation between nocturnal decline in blood pressure and mortality. The Ohasama Study. Am J Hypertens 10: 1201-1207.

16. Ohkubo T, Hozawa A, Yamaguchi J, Kikuya M, Ohmori K, et al. (2002) Prognostic significance of the nocturnal decline in blood pressure in individuals with and without high 24-h blood pressure: the Ohasama study. J Hypertens 20: $2183-2189$

17. Grossman AB (2010) Clinical Review\#: The diagnosis and management of central hypoadrenalism. J Clin Endocrinol Metab 95: 4855-4863.
18. Speiser PW, Azziz R, Baskin LS, Ghizzoni L, Hensle TW, et al. (2010) Congenital adrenal hyperplasia due to steroid 21-hydroxylase deficiency: an Endocrine Society clinical practice guideline. J Clin Endocrinol Metab 95: 41334160.

19. Esteban NV, Loughlin T, Yergey AL, Zawadzki JK, Booth JD, et al. (1991) Daily cortisol production rate in man determined by stable isotope dilution/mass spectrometry. J Clin Endocrinol Metab 72: 39-45.

20. Crown A, Lightman S (2005) Management of patients with glucocorticoid deficiency. Nat Clin Pract Endocrinol Metab 1: 62-63.

21. Crown A, Lightman S (2005) Why is the management of glucocorticoid deficiency still controversial: a review of the literature. Clin Endocrinol (Oxf) 63: 483-492.

22. Merke DP (2008) Approach to the adult with congenital adrenal hyperplasia due to 21-hydroxylase deficiency. J Clin Endocrinol Metab 93: 653-660.

23. Azziz R, Dewailly D, Owerbach D (1994) Clinical review 56: Nonclassic adrenal hyperplasia: current concepts. J Clin Endocrinol Metab 78: 810-815.

24. Joint LWPES/ESPE CAH Working Group (2002) Consensus statement on 21-hydroxylase deficiency from the Lawson Wilkins Pediatric Endocrine Society and the European Society for Paediatric Endocrinology. J Clin Endocrinol Metab 87: 4048-4053.

25. German A, Suraiya S, Tenenbaum-Rakover Y, Koren I, Pillar G, et al. (2008) Control of childhood congenital adrenal hyperplasia and sleep activity and quality with morning or evening glucocorticoid therapy. J Clin Endocrinol Metab 93: $4707-4710$

26. Charmandari E, Hindmarsh PC, Johnston A, Brook CG (2001) Congenital adrenal hyperplasia due to 21-hydroxylase deficiency: alterations in cortisol pharmacokinetics at puberty. J Clin Endocrinol Metab 86: 2701-2708.

27. Ross RJ, Rostami-Hodjegan A (2005) Timing and type of glucocorticoid replacement in adult congenital adrenal hyperplasia. Horm Res 64 Suppl 2 . $67-70$

28. Moeller H (1985) Chronopharmacology of hydrocortisone and 9 alphafluorhydrocortisone in the treatment for congenital adrenal hyperplasia. Eur $\mathrm{J}$ Pediatr 144: 370-373.

29. Palczewska I, Niedzwiecka Z (2001) somatic development indices in children and youth of Warsaw. Med Wieku Rozwoj 5: 18-118.

30. Roche EF, Charmandari E, Dattani MT, Hindmarsh PC (2003) Blood pressure in children and adolescents with congenital adrenal hyperplasia (21-hydroxylase deficiency): a preliminary report. Clin Endocrinol (Oxf) 58: 589-596.

31. Völkl TM, Simm D, Dötsch J, Rascher W, Dörr HG (2006) Altered 24-hou blood pressure profiles in children and adolescents with classical congenital adrenal hyperplasia due to 21-hydroxylase deficiency. J Clin Endocrinol Metab 91: 4888-4895. 